\title{
Amino acid composition of droughtmaster beef at various beef cuts
}

\author{
Zainal Samicho, Siti Roha Ab Mutalib ${ }^{*}$, Noriham Abdullah
}

Department of Food Technology, Faculty of Applied Sciences, Universiti Teknologi MARA, 40450 Shah Alam, Malaysia;

*Corresponding Author: siti roha23@yahoo.com.my

Received 2013

\begin{abstract}
A total of 14 parts of beef cuts were used to determine the amino acid composition in droughtmaster beef. Drought-master beef is a cross breed tropical cattle with $50 \%$ Shorthorn and $50 \%$ Brahman cattle. The most abundant type of amino acid in drought-master beef was glutamic acid, followed by aspartic acid, lysine, leucine and arginine. The flank cut of beef was found to contain higher amount of total amino acids followed by top side and short rib cut of beef. The amino acid composition of drought-master beef indicates that beef is a good source of dietary protein for human.
\end{abstract}

Keywords: Amino Acid; Droughtmaster Beef; Beef Cut and Acid hydrolysis

\section{INTRODUCTION}

Beef has an excellent nutritional quality because it has proteins of high biological value, it is rich in vitamin contents, especially B-complex, and it is associated to a high mineral content, especially iron, in high bioavailability form [1]. Beef contains all the amino acids in about the right proportions required by humans [2]. Many studies deal with the opportunities of enhancing the beneficial fatty acids (increasing the $n-3$ polyunsaturated fatty acids and conjugated linoleic acids (CLA) and reducing saturated fatty acids (SFA)) in beef, but little is known about the alteration of other nutritional ingredients (amino acid composition and mineral content) in beef $[3,4]$.

Meat and meat products are good sources of protein for human consumption. These proteins are well balanced in amino acid and contain all essential amino acid that human cannot be synthesized. However, because of health effect some people cannot consumed meat. According to [5], several essential amino acids such as leucine and phenylalanine and non essential amino acid such as arginine and glutamine, have shown to directly stimulate insulin secretion from pancreatic beta-cells, so as it has beneficial therapeutic effect in patient with type 2 diabetes.

Droughtmaster beef is mixed tropical cattle 50\% Shorthorn and 50\% Brahman cattle. It can stand with hot and humid conditions resist to tick and good reproduction. Up to date, there is no amino acid study on beef species droughtmaster have been done and there are few studies focused on amino acid in different beef cut. Therefore, the aim of the experiments was to establish different amino acid content in different droughtmaster beef cut.

\section{MATERIAL AND METHODS}

Amino acid kit (AccQ Tag) was purchased from Waters. All other chemicals were purchased from Sigma. Beef were purchased from Rantau Panjang, Selangor, Malaysia.

\subsection{Preparation of Beef}

14 parts of beef cut were used in this study. The parts are neck clod, short rib, shin, flank, rump, brisket, thick flank, top side, silver side, thin rib, thick rib, chuck blade, sirloin and leg.

\subsection{Acid Hydrolysis}

The amino acid composition of the samples was analyzed by digesting the samples for 24 hour at $110^{\circ} \mathrm{C}$ in an oven with $5 \mathrm{ml}$ of $6 \mathrm{~N} \mathrm{HCl}$ in sealed glass test tubes. The aliquot of the hydrolysate was taken and $0.4 \mathrm{ml}$ AABA (alpha amino butyric acid $(50 \mu \mathrm{mol} \mathrm{ml}-1))$ was added to it as the internal standard. Then $100 \mathrm{ml}$ of deionized water was added to the aliquot. This aliquot was then filtered using whatman filter paper No.1 followed by a syringe filter.

\subsection{Derivatization of Amino Acids with 6-aminoquinolyl-N-hydroxysuccinimidy I Carbamate (AQC)}

A cleaned syringe was used to deliver $10 \mu \mathrm{L}$ filtrate of 
acid hydrolysis to the bottom of a cleaned $6 \times 50 \mathrm{~mm}$ sample tube. A $70 \mu \mathrm{L}$ of AccQ $\bullet$ Fluor Borate Buffer (Reagent 1) was then added to the sample tube by using a micropipette. The sample tube was vortexed briefly prior to adding of $20 \mu \mathrm{L}$ of reconstituted AccQ $•$ Fluor Reagent to the sample tube. After vortexed for several seconds, the sample tube was let to stand for 1 minute at room temperature.

\subsection{Preparation of Internal Standard and Calibration Standard}

The internal standard, AABA stock solution was used to prepare the calibration standard. To prepare a 50 $\mu \mathrm{mol} / \mathrm{mL}$ internal standard stock solution, $0.258 \mathrm{~g}$ AABA was added to $50 \mathrm{~mL} 0.1 \mathrm{~N} \mathrm{HCl}$. Prior to the preparation of the calibration standard with an internal standard, 1 $\mathrm{mL}$ of $50 \mu \mathrm{mol} / \mathrm{mL}$ internal standard stock solution was transferred to a cleaned $20 \mathrm{~mL}$ volumetric flask. The solution was then made up to $20 \mathrm{~mL}$ with $0.1 \mathrm{~N} \mathrm{HCl}$ until the final concentration of the internal standard solution was $2.5 \mu \mathrm{mol} / \mathrm{mL}$. The calibration standard consisted of 1:1 (v/v) mixture of Pierce $\mathrm{H}$ amino acid standard (which contained $2.5 \mu \mathrm{mol} / \mathrm{mL}$ of each amino acid standard, except for $1.25 \mu \mathrm{mol} / \mathrm{mL}$ of cystine) and a $2.5 \mu \mathrm{mol} / \mathrm{mL}$ of AABA. Typically, a calibration standard with an internal standard was prepared by combined $80 \mu \mathrm{L} 2.5$ $\mu \mathrm{mol} / \mathrm{mL}$ AABA with $80 \mu \mathrm{L}$ Pierce $\mathrm{H}$ and then was made up with $840 \mu \mathrm{L}$ deionized water in a $1000 \mu \mathrm{L}$ cleaned vial. A volume of $10 \mu \mathrm{L}$ calibration standard (contains of 2 nmol of each standard amino acid components) was transferred from the 1:1 (v/v) mixture of Pierce $\mathrm{H}$ amino acid standard, and $2.5 \mu \mathrm{mol} / \mathrm{mL}$ of AABA was placed in a derivatisation tube and carried through the same derivatisation procedure as mentioned earlier.

\subsection{HPLC Analysis of Amino Acids}

The Waters AccQ•Tag amino acid analysis method requires a fluorescence detector. The excitation wavelength was $285 \mathrm{~nm}$, the emission wavelength was 354 $\mathrm{nm}$, filter and gain set were 1.5 second and 10 , respectively. Eluent A and Eluent B were AccQ•Tag concentrate and $60 \%$ acetonitrile: water, respectively. The column temperature was set at $37^{\circ} \mathrm{C}$. The Column (Waters AccQ $\bullet$ Tag) was first conditioned with Eluent B at 1 $\mathrm{mL} / \mathrm{min}$ flow rate for 5 minutes. This was followed by equilibrating the column in $100 \%$ AccQ $\cdot$ Tag Eluent A for 9 minutes at the same flow rate. Consistent period of the equilibration was kept for all the analysis. A blank was carried out before each analysis to determine baseline performance. The gradient shown in Table 1 was used in the process of analyzing amino acids using HPLC. The total time between injections to end of the analysis was 50 minutes.
Table 1. Gradient table for amino acids analysis using HPLC.

\begin{tabular}{cccc}
\hline Time (min) & Flow rate (mL/min) & $\mathbf{\% A}$ & $\mathbf{\% B}$ \\
\hline Initial & 1.0 & 100.0 & 0.0 \\
0.50 & 1.0 & 98.0 & 2.0 \\
15.0 & 1.0 & 90.0 & 10.0 \\
19.0 & 1.0 & 87.0 & 13.0 \\
32.0 & 1.0 & 65.0 & 35.0 \\
33.0 & 1.0 & 65.0 & 35.0 \\
34.0 & 1.0 & 0.0 & 100.0 \\
37.0 & 1.0 & 0.0 & 100.0 \\
38.0 & 1.0 & 100.0 & 0.0 \\
50.0 & 1.0 & 100.0 & 0.0 \\
\hline
\end{tabular}

\subsection{Statistical Analysis}

All data were expressed as mean \pm standard deviation. Data were analysed using one-way ANOVA using SPSS 15.0. Duncan's multiple-range test was used to determine the difference between means. A significant difference was considered at the level of $p<0.05$.

\section{RESULTS AND DISCUSSION}

Droughtmaster beef was used in this study and it was divided into 14 parts of beef cut. The method used in this study only allowed analysis of 17 amino acids. These amino acids were arginine, lysine, valine, threonine, leucine, tyrosine, histidine, isoleucine, phenylalanine, methionine, cysteine, glycine, proline, alanine, glutamic acid, aspartic acid and serine. Upon acid hydrolysis, asparagines and glutamine could be hydrolyzed to aspartic acid and glutamic acid, respectively. Thus, the content of aspartic acid represents the total content of asparagines and aspartic acid, and the same applies to glutamic acid. Tryptophan is destroyed upon acid hydrolysis, thus was not measured in this study.

Table 2 shows the amino acids content in different beef cuts. The amino acid content is presented as gram amino acid/ 100 gram total amino acids. The major components of amino acid were glutamic acid, followed by aspartic acid, lysine, leucine and arginine. There was significant difference at the $5 \%$ level in the amino acid contents among 14 parts of beef cuts tested.

The content of aspartic acid, serine, glutamic acid, arginine, threonine, valine, methionine, lysine, isoleucine, leucine and phenylalanine was significantly higher in flank cut of beef part. This finding was in agreement with studied done by [6] on the amino acid content in fresh and cooked beef cuts. Furthermore, the content of certain amino acid such as tyrosine, alanine and histidine were significantly higher in top side of beef cut. On the other hand, proline and glycine was significantly higher in short rib and cysteine was higher in thick flank of beef cut. 


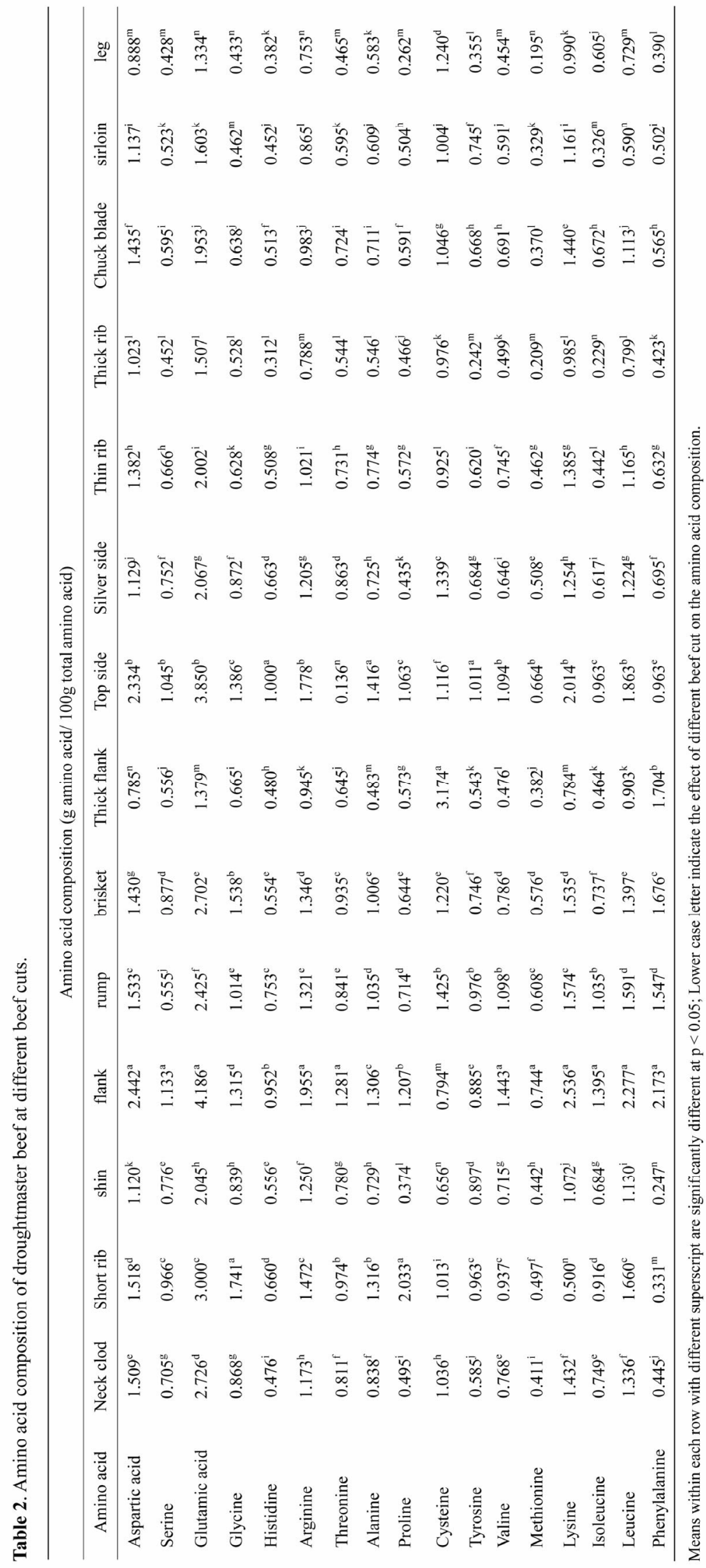


In summary there is no general pattern correlation between the beef cut and amino acid content in beef. When comparing between different parts of beef cut, the amino acid content in beef cut were almost similar. The most abundant amino acid in beef (in decreasing order) was glutamic acid, aspartic acid, lysine, leucine, cysteine, arginine, glycine and phenylalanine. However, histidine and methionine were present in much lower amount which was in agreement with [6]. The essential amino acid comprised more than $40 \%$ of total amino acid content of beef cut. The total amino acid content for neck clod, short rib, shin, flank, rump, brisket, thick flank, top side, silver side, thin rib, thick rib, chuck blade, sirloin and leg were 16.36, 20.50, 14.31, 28.02, 19.95, 19.71, $14.94,23.70,15.68,14.26,10.33,14.71,12.00$ and 10.49 ( $\mathrm{g} / 100 \mathrm{~g}$ of sample) respectively.

When comparing this research finding with previous studied done by [6], there is slightly different in the amount of amino acid content. The rationale for this may be due to different species of beef, animal age, effect of feeding high or low amino acid compound, processing [7], slaughter weight and gender [8].

With regards the uses of amino acid, several essential amino acids such as leucine and phenylalanine and non essential amino acid such as arginine and glutamine, have shown to directly stimulate insulin secretion from pancreatic beta-cells, so as it has beneficial therapeutic effect in patient with type 2 diabetes [5] and [9]. According to [10], $1 \mathrm{mmol} / \mathrm{kg}$ lean body mass leucine plus $25 \mathrm{~g}$ glucose will synergistically stimulate insulin secretion. Another function of leucine is to stimulate protein synthesis, inhibit protein degradation and nutrient signaling molecule [10]. Amino acids are also important in healing process. Deficiency in essential amino acids may hinder healing recovery process [11]. Leucine promotes the healing of bones, skin and muscle tissue. Isoleucine is necessary for haemoglobin formation, stabilizing and regulating blood sugar and energy. Glycine, which is one of the major components of human skin collagen, together with other essential amino acids such as alanine form a polypeptide that will promote regrowth and tissue healing [12].

\section{CONCLUSIONS}

The amino acid composition of beef cut showed that it is a good source of dietary amino acid. With regard to its nutritional value, beef provides all the essential amino acids needed by human. The essential amino acid cannot be manufactured by human bodies but can be obtained from food. Flank cut of beef is found rich in leucine which is type of essential amino acid that can stimulate insulin secretion.

\section{ACKNOWLEDGEMENTS}

Special thanks to Ministry of Higher Education, Malaysia for funding this project under Fundamental Research Grant Scheme [600-RMI/ ST/FRGS/Fst(32/2010)].

\section{REFERENCES}

[1] Saucier, L.(1999) Meat safety: Challenges for the future. Nutrition Abstracts and Reviews (Series A), 69, 705-708.

[2] Pensel, N.(1998) The future of meat in human diets. Nutrition Abstracts and Reviews (Series A), 68, 1-4.

[3] Scollan, N., Hocquette, J.F., Nuernberg, K. and Dannenberger, D. (2006) Innovation in Beef Production Systems that Enhance the Nutritional and Health Value of Beef Lipids and Their Relationship with Meat Quality. Meat Science, 70, 17-33. doi.org/10.1016/j.meatsci.2006.05.002

[4] Hollo, G., Nuernberg, K., and Hollo, I. (2007) Effect of feeding on the composition of longissmus muscle of hungarian grey and the holsteh friesian bulls. Arch Tierz Dummersturf50, 6, 575-586.

[5] Bjoern, A.M., Schrader, H. and Ritter, D.R. (2010) Selective amino acid deficiency in patients with impaired glucose tolerance and type 2 diabetes. Regulatory Peptide, 160, 75-80. doi:10.1016/j.regpep.2009.08.001

[6] Greenwood, D.A., Kraybill, H.R., and B.S, S. (1951) Amino acid composition of fresh and cooked beef cuts. Journal of Bioligical Chemistry, 36, 23-28.

[7] Lawrie, R.A. (1998) Lawrie's Meat Science (6th ed.). Cambridge England: Woodhead Publishing Limited.

[8] Hollo, G., Csapo, J., Szues, E., Tozser, J., Repa, I. and Hollo, I. (2001) Influence of Breed, Slaughter weight and Gender on Chemical Composition of Beef. Part 1. Amino Acid Profile and Biological V alue of Proteins. Asian-Australia Journal Animal Science, 14, 1555-1559.

[9] Soucy, J. and Blanc, J.L. (1998) The Effects of a beef and fish meal on plasma mmino acids, Insulin and glucagon level. Nutrition Research, 19, 17-24. doi:10.1016/S0271-5317(98)00171-7

[10] Kalogeropoulou, D., LaFave, L., Schweim, K., Mary C, G. and Frank, Q.N. (2008) Leucine, when ingested with glucose, synergistically stimulates insulin secretion and lowers blood glucose. Metabolism Clinical and Experimental, 57, 1747-1752. doi:10.1016/j.metabol.2008.09.001

[11] Mat Jais, A.M., McCulloh, R. and Croft, K. (1994) Fatty acid and amino acid composition in haruan as a potential role in wound healing. General Pharmacology, 25, 947-950. doi:10.1016/0306-3623(94)90101-5

[12] Witte, M.B., Thornton, F.J., Tantry, U. and Barbul, A. (2002) L- Arginine supplementation enhances diabetic wound healing; involvement of the nitric oxide synthase and arginase pathways. Metabolism, 51, 1269-1273. doi:10.1053/meta.2002.35185 